This item was submitted to Loughborough's Research Repository by the author.

Items in Figshare are protected by copyright, with all rights reserved, unless otherwise indicated.

Achieving consistent performance in a complex whole body movement: the Tkatchev on high bar

PLEASE CITE THE PUBLISHED VERSION

http://dx.doi.org/10.1016/j.humov.2011.08.009

PUBLISHER

(C) Elsevier B.V.

VERSION

AM (Accepted Manuscript)

LICENCE

CC BY-NC-ND 4.0

REPOSITORY RECORD

Hiley, Michael J., and Maurice R. Yeadon. 2019. "Achieving Consistent Performance in a Complex Whole Body Movement: The Tkatchev on High Bar”. figshare. https://hdl.handle.net/2134/11486. 
This item was submitted to Loughborough's Institutional Repository (https://dspace.lboro.ac.uk/) by the author and is made available under the following Creative Commons Licence conditions.

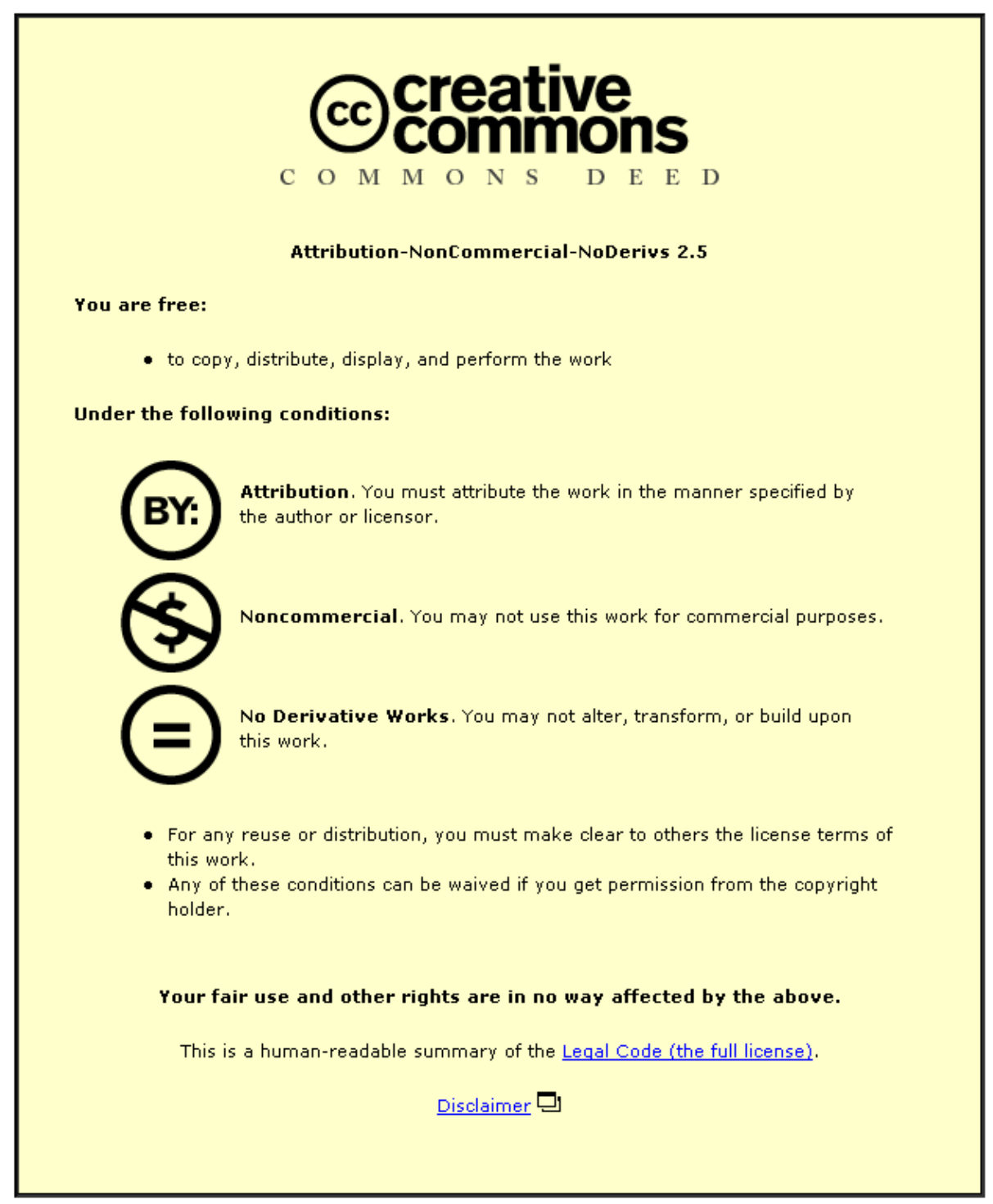

For the full text of this licence, please go to: http://creativecommons.org/licenses/by-nc-nd/2.5/ 


\title{
Achieving consistent performance in a complex whole body movement: the Tkatchev on high bar
}

\author{
Michael J. Hiley and Maurice R. Yeadon \\ School of Sport, Exercise and Health Sciences, Loughborough University, Leicestershire, LE11 3TU, UK
}

\begin{abstract}
If the magnitude of timing and angle variability in whole body coordinated movements were known, this would allow more realistic levels of variability to be included within optimisations of technique. The aim of this study was to determine technique for improved consistency of performance of the Tkatchev release and regrasp on high bar, whilst incorporating realistic levels of coordination precision. The effect of gymnast strength and flexibility on consistency of performance was also investigated. Twenty trials (10 successful and 10 unsuccessful) by one national gymnast were recorded using an automatic motion capture system and were analysed to determine variability in coordination of the giant circle technique prior to release. The standard deviation in the hip and shoulder angles and timings at four key instants in the gymnast's performances were $2.3^{\circ}$ and $12 \mathrm{~ms}$. A gymnast - high bar simulation model was used to optimise the technique in the giant circle to maximise the success percentage for which the gymnast could release and regrasp the bar with coordination variability introduced into each simulated technique. When the optimal solution was perturbed randomly in 1000 simulations to the level seen in the gymnast performances $69 \%$ produced a successful performance compared with only $17 \%$ for the gymnast. An increase in strength (by $25 \%$ ) and a reduction in variability (by $25 \%$ ) lead to improved consistency ( $91 \%$ success rate). Flexibility did not appear to play a role as none of the optimisations approached the bounds set by the gymnast's performances.
\end{abstract}

Keywords: simulation, variability, strength, flexibility, optimisation, gymnastics

\section{INTRODUCTION}

The Tkatchev release and regrasp on high bar is performed from a backwards rotating giant circle. During release the direction of rotation must be reversed so that the gymnast rotates forwards in flight while travelling backwards over the bar to regrasp (Figure 1). The gymnast adopts a straddled configuration in flight to reduce the moment of inertia about the lateral axis through the mass centre. Previous experimental research has looked at the mechanical descriptors of the preceding giant circles and the release parameters of the Tkatchev (Gervais and Tally, 1993; Brüggemann et al., 1994). Simulation models have been used to investigate the differences between successful and unsuccessful performances, with attempts to identify how missed regrasps could be corrected (Holvoet et al., 2002; Hiley et al., 2007). Holvoet et al. (2002) demonstrated that an unsuccessful Tkatchev could have been caught had the gymnast released the bar earlier than in the actual performance. In contrast, Hiley et al. (2007) found that for another gymnast earlier release in unsuccessful performances would not have enabled the bar to be regrasped. In order to improve the consistency of performance (i.e. increase the percentage of successful attempts) this gymnast would need to change his technique. 


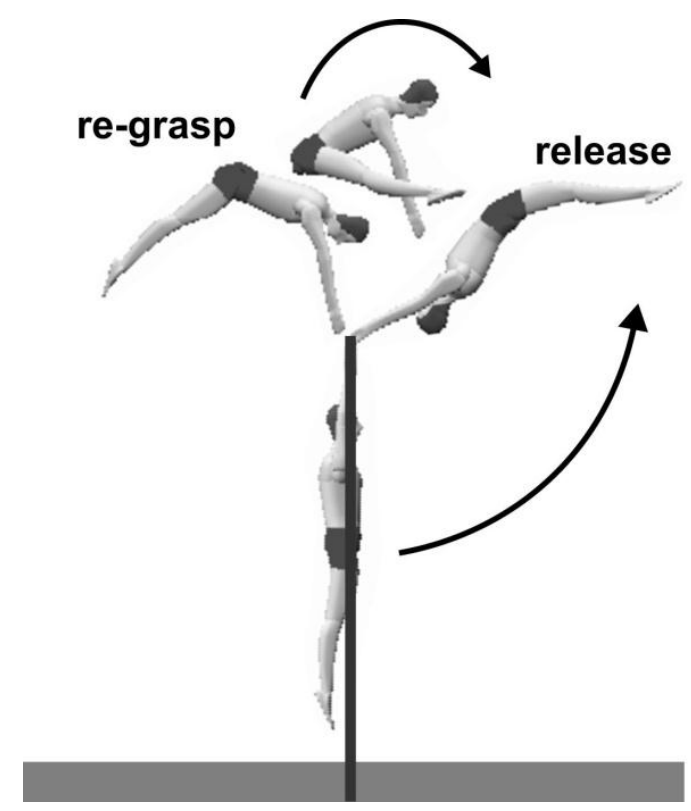

Figure 1. The Tkatchev release and regrasp on high bar.

When a gymnast performs the same skill a number of times it might be expected that he is attempting to use the same technique. However, it is also to be expected that within each attempt there will be some variability in the technique used (Newell and Corcos, 1993; Wilson et al., 2007; Wilson et al., 2008). The term coordination precision will be used here to refer to the level of variability in the timing and angles of the movement when the gymnast performs the same skill a number of times. Since it is not possible for the gymnast to coordinate the skill precisely (timings and angles) each time, his technique should have developed to cope with the level of variability. A gymnast's technique should therefore be robust so that it can produce similar results (e.g. production of linear and angular momentum) despite the level of variability present (i.e. the gymnast's coordination precision). If a gymnast's technique is to be optimised to improve consistency of performance, it is necessary to establish the magnitude of the variability present in the gymnast's technique so that it can be incorporated in the optimisation process and so that the robustness of the new technique can be assessed.

A successful Tkatchev is one in which the gymnast regrasps the bar. For a successful performance the gymnast must have sufficient linear and angular momentum at release to place the gymnast in a position to regrasp the bar at the end of the flight phase. Hiley et al. (2007) determined the release timing window of the preceding giant circle for 10 successful and 10 unsuccessful Tkatchev attempts. The release timing window was defined as the interval for which the gymnast had sufficient linear and angular momentum for a successful performance. In order for the gymnast to regrasp the bar a sufficiently large release window is required since otherwise the gymnast will be unable to time the release accurately. The release windows for the successful trials ranged from $9-74 \mathrm{~ms}$ (mean $29 \pm 21 \mathrm{~ms}$ ) whereas the release windows for the unsuccessful trials, as might be expected, were much smaller (mean $3 \pm 4 \mathrm{~ms}$ ). 
Many competitive gymnasts are able to perform release and regrasp skills on high bar with a success rate higher than $90 \%$ while other gymnasts are unable to achieve such success rates. The reasons for this may include:

- $\quad$ use of inappropriate technique

- lack of coordination precision

- lack of strength

- lack of flexibility

The aim of this study is to investigate to what extent changes in technique, strength and flexibility can improve the consistency of the Tkatchev performance.

\section{METHOD}

An initial analysis of the data collected by Hiley et al. (2007) was carried out to determine the variability in coordination (timing and angle) with which the gymnast performed actions at the hip and shoulder joints during giant circles prior to a Tkatchev release and regrasp. Subsequently a gymnast - high bar simulation model (Hiley and Yeadon, 2003) was used to optimise the technique in the backward giant circle prior to release in order to increase the consistency of performance. The optimisation of technique included consideration of the gymnast's coordination precision (variability in timing and angles), based on the initial analysis.

\subsection{Data analysis}

The coordination precision was determined from the kinematic data collected by Hiley et al. (2007) from 20 performances of the Tkatchev. One senior male gymnast competing at national level (mass $=64 \mathrm{~kg}$, height $1.63 \mathrm{~m}$ ) performed 60 Tkatchev release and regrasps which were recorded using nine Vicon M2 cameras operating at $100 \mathrm{~Hz}$. Spherical reflective markers of $25 \mathrm{~mm}$ diameter were attached to the lateral side of the wrist, elbow, shoulder, hip, knee and ankle joint centres and toes on each side of the body. Offset measurements from each marker centre to the adjacent joint centre were recorded for subsequent location of the joint centres. Additional markers were attached to each side of the gymnast's head (above the ear) and to the centre of the high bar. Prior to data collection a volume centred on the high bar spanning $2 \mathrm{~m} \times 5 \mathrm{~m} \times 5 \mathrm{~m}$ was wand calibrated using the Vicon motion analysis system. The 10 successful trials and the 10 nearest misses of the bar were chosen for initial analysis. Three-dimensional marker coordinates were reconstructed and joint centres were calculated using the measured offsets from which arm orientation and joint configuration angles were calculated (Yeadon, 1990b) and quintic splines (Wood and Jennings, 1979) were used to fit joint angle time histories so that the data could be interpolated.

To calculate the timing and angle variability of the gymnast the instants of maximum and minimum hip and shoulder flexion and extension angles (Figure 2) were determined from the quintic splines (i.e. determine the start time, end time and magnitude of a joint flexion/extension). Time zero corresponded to a whole body rotation angle (the angle between the line joining the neutral bar location to the gymnast mass centre and the upward vertical) of $120^{\circ}$ after the vertical. The time taken to rotate to the mean release angle $\left(305^{\circ}\right)$ was also recorded. The mean and standard deviation were calculated for each measure. 


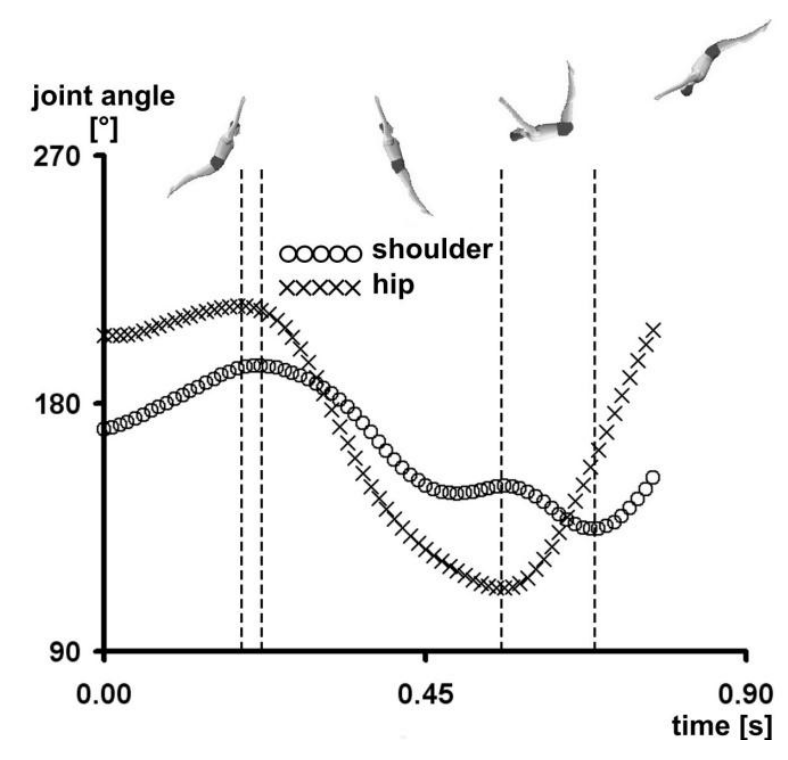

Figure 2. Joint angle time histories of the hip and shoulder with the maximum and minimum flexion/extension angles indicated along with a graphical representation of the gymnast.

\subsection{Simulation model}

A four segment simulation model, which included damped linear springs for the elastic structures of the gymnast and high bar, was used (Hiley and Yeadon, 2005). Input to the model comprised the initial conditions (initial spring displacements, model configuration and velocities) and the joint angle time histories of the shoulder, hip and knee in the form of piecewise quintic functions (Hiley and Yeadon, 2003). In order to define a joint angle time history the start time, end time and magnitude of each angle change must be specified. Output from the model comprised the whole body rotation angle and the linear and angular momentum about the mass centre of the model. The model incorporated subject-specific inertia data (Yeadon, 1990a). To obtain model parameters which could not be calculated directly (e.g. spring stiffness and damping coefficients) a matching procedure was carried out as described in Hiley and Yeadon (2005). A simulation was run with the matched parameters to assess the closeness of fit to the recorded data. Strength characteristics were scaled from data on an elite male gymnast (King and Yeadon, 2002; King et al., 2009) so that the torques in the matching simulation lay within these limits.

\subsection{Optimisation}

The parameters defining the joint angle time histories of the shoulder, hip, and knee were varied in order to maximise the number of successful performances produced when the technique was randomly perturbed to the level of the variability measured in the gymnast performances (i.e. to increase the consistency of performance). A successful performance was one that produced a sufficiently large release window (described below). The parameters included the start and end time of each joint flexion/extension and the magnitude of the angle change (Hiley and Yeadon, 2003). Simulations were penalised if the joint angle time histories resulted in joint torques which exceeded those determined from the subject-specific strength characteristics. The mechanics of the Tkatchev requires a joint anglular velocity at the hip and/or shoulder at release (Hiley et al., 2007). The hip extension and shoulder flexion angles were not allowed to exceed the maximum values observed in 
the recorded performances (hip $=230^{\circ}$, shoulder $=210^{\circ}$, where $180^{\circ}$ corresponds to a straight body position with the arms overhead). As the quintic functions used have zero velocity at the end points, this would ensure that the anatomical limits could not be exceeded after release.

The release window was defined as the period of time for which the model possessed normalised angular momentum (Kerwin et al., 1990) within the range of the 10 successful trials $\pm 10 \%$ of that range (Hiley et al., 2007) and linear momentum to place the model in a position to regrasp the bar. In order for the model to be within successful catching distance of the bar, the mass centre had to lie within a sector defined by the range of actual catch positions and those that would be anatomically feasible. The path of the mass centre in flight was calculated using the mass centre location and velocity at release and equations of motion under constant acceleration. The release window was allowed to start before and end after the actual release time of the trial so long as the above conditions were satisfied.

To investigate the effect of variability in the timing and angles of the shoulder and hip actions, perturbations were added to the start time, end time and angle parameters of the joint angle time histories with the standard deviation levels of 12 $\mathrm{ms}$ and $2.3^{\circ}$ obtained from the analysis of the 20 gymnast performances. A random number generator with a normal distribution was used to add variability to the parameters of the joint angle time histories to the specified level. For each set of joint angle time history parameters produced by the genetic optimisation algorithm (Carroll, 2001), 500 randomly perturbed simulations were performed. From Hiley et al. (2007) the critical size of release window for a successful Tkatchev was approximately $10 \mathrm{~ms}$. Only one successful trial of the gymnast had a window less than $10 \mathrm{~ms}(9 \mathrm{~ms})$ and only one unsuccessful trial had a window greater than $10 \mathrm{~ms}$ (12 ms). The perturbed simulations were given a score based on the size of the release window produced:

$$
\begin{aligned}
& \text { score }=0, \text { if release window }=0 \mathrm{~ms} \\
& \text { score }=1, \text { if } 0 \mathrm{~ms}<\text { release window }<10 \mathrm{~ms} \\
& \text { score }=2, \text { if release window } \geq 10 \mathrm{~ms}
\end{aligned}
$$

The sum of scores for the 500 simulations was maximised using a parallelised genetic optimisation algorithm (Carroll, 2001; van Soest and Casius, 2003). The optimal solution was subsequently used to produce 1000 randomly perturbed simulations in order to determine the percentage success.

To investigate the effect of the level of the timing and angle variability on consistency the optimisation was repeated with the level of the perturbations reduced by $25 \%$ (i.e. to a standard deviation of $9 \mathrm{~ms}$ and $1.7^{\circ}$ ). To investigate the effect of strength on consistency the above two optimisations were repeated with the joint torques calculated from the joint angle - angular velocity - torque profiles increased by $25 \%$. One further optimisation was performed which maximised the release window without considering variability (i.e. no perturbations added to the joint angle time history parameters). The solution was also run 1000 times with random perturbations to demonstrate the effect of not including variability within the optimisation. All of the above optimisations were started with the same upper and lower bounds for each joint angle parameter, no initial technique was required as a starting point. 


\section{RESULTS}

The 20 analysed backward giant circles prior to release for the Tkatchev were very consistent in terms of duration and timing of the hip and shoulder actions. The mean duration of the preceding swing, from a rotation angle of $120^{\circ}$ through to the mean release angle of $305^{\circ}$, was $695 \pm 9 \mathrm{~ms}$ (Table 1). The mean standard deviation for the times at maximum and minimum joint angles for all 20 performances was $12 \mathrm{~ms}$. The data for each time were found to be normally distributed using the Kolmorgorov-Smirnov test. When the data from each trial were normalised to duration the mean standard deviation for the times at maximum and minimum joint angles was still equivalent to $12 \mathrm{~ms}$. The mean standard deviation for the joint angles at the same instants was $2.3^{\circ}$ (Table 1 ).

Table 1. Mean angle and times ( \pm standard deviation) at key points within the joint angle time history of the preceding giant circle

\begin{tabular}{l|c|c|c|c|c|c|c|c|c}
\hline \multicolumn{1}{c|}{ Group } & \multicolumn{9}{|c|}{ Mean angles at } \\
& $\begin{array}{c}\text { Max hip } \\
\text { angle } \\
{\left[^{\circ}\right]}\end{array}$ & $\begin{array}{c}\text { Max } \\
\text { shld } \\
\text { angle } \\
{\left[^{\circ}\right]}\end{array}$ & $\begin{array}{c}\text { Min hip } \\
\text { angle } \\
{\left[^{\circ}\right]}\end{array}$ & $\begin{array}{c}\text { Min } \\
\text { shld } \\
\text { angle } \\
{\left[^{\circ}\right]}\end{array}$ & $\begin{array}{c}\text { Max hip } \\
\text { angle } \\
{[\mathrm{ms}]}\end{array}$ & $\begin{array}{c}\text { Max shld } \\
\text { angle } \\
{[\mathrm{ms}]}\end{array}$ & $\begin{array}{c}\text { Min hip } \\
\text { angle } \\
{[\mathrm{ms}]}\end{array}$ & $\begin{array}{c}\text { Min shld } \\
\text { angle } \\
{[\mathrm{ms}]}\end{array}$ & $\begin{array}{c}\text { Release } \\
{[\mathrm{ms}]}\end{array}$ \\
\hline $\begin{array}{l}\text { Successful } \\
(\mathrm{n}=10)\end{array}$ & $216^{\circ} \pm 3$ & $193^{\circ} \pm 2$ & $114^{\circ} \pm 2$ & $133^{\circ} \pm 3$ & $118 \pm 9$ & $152 \pm 6$ & $481 \pm 11$ & $609 \pm 14$ & $699 \pm 7$ \\
$\begin{array}{l}\text { Unsuccessful } \\
(\mathrm{n}=10)\end{array}$ & $214^{\circ} \pm 2$ & $194^{\circ} \pm 2$ & $115^{\circ} \pm 2$ & $133^{\circ} \pm 1$ & $118 \pm 10$ & $162 \pm 20$ & $484 \pm 10$ & $611 \pm 13$ & $690 \pm 8$ \\
$\begin{array}{l}\text { Combined } \\
(\mathrm{n}=20)\end{array}$ & $215^{\circ} \pm 3$ & $193^{\circ} \pm 2$ & $115^{\circ} \pm 2$ & $133^{\circ} \pm 2$ & $118 \pm 9$ & $157 \pm 15$ & $483 \pm 11$ & $610 \pm 13$ & $695 \pm 9$ \\
\hline
\end{tabular}

The matching simulation was able to estimate the whole body rotation angle to $3^{\circ}$, the bar displacements to $0.01 \mathrm{~m}$ and the joint angle time histories to $2^{\circ}$ (root mean squared differences) of the actual performance (Figure 3). The release window for the matching simulation was $24 \mathrm{~ms}$.

The results of the optimisation to investigate the effect of variability on performance showed that at a perturbation level with a standard deviation of $12 \mathrm{~ms}$ for the timings and $2.3^{\circ}$ for the angles, the mean release window from 1000 perturbed simulations (Table 2) was $34 \mathrm{~ms}$, which was larger than that obtained from the 20 analysed gymnast performances $(15 \mathrm{~ms})$. More importantly there was an improvement in consistency (i.e. larger percentage of successful simulations); when the optimal simulation was run 1000 times and randomly perturbed, $69 \%$ of simulations produced a sufficiently large release window to result in a successful performance (i.e. $>10 \mathrm{~ms}$ ), compared only $17 \%$ (Table 2 ) if all 60 of the gymnast trials are considered (assuming the remaining 40 unsuccessful trials not analysed had release windows comparable with the analysed trials). While the criterion was for the release window to be greater than $10 \mathrm{~ms}$ most of the $69 \%$ successes had release windows greater than the mean window $(29 \mathrm{~ms})$ of the 10 successful Tkatchevs. 


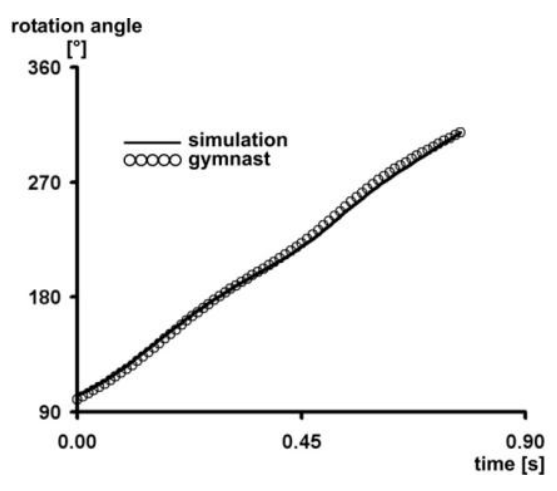

a

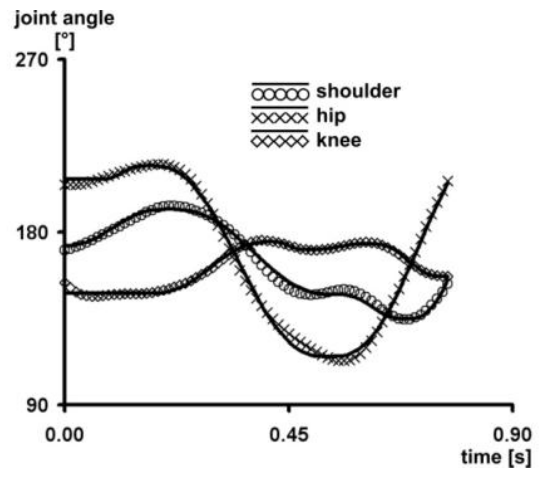

C

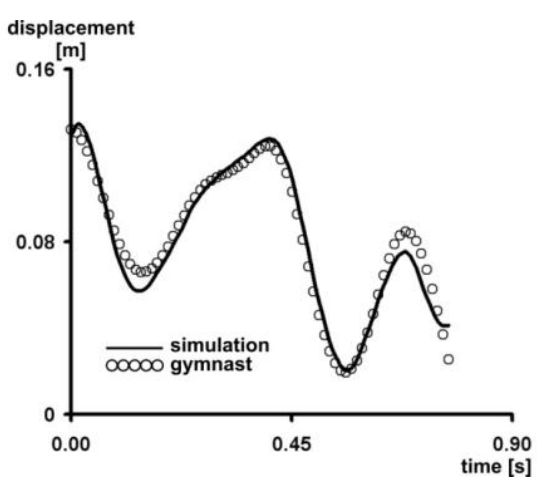

b

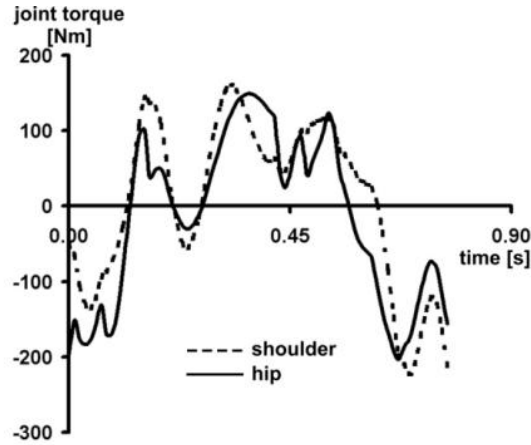

d

Figure 3. Matching simulation (a) rotation angle, (b) bar displacement, (c) joint angle time histories and (d) joint torques.

Table 2. Release windows obtained from the optimisations with different variability and joint torque constraints, and the analysed gymnast performances

\begin{tabular}{|c|c|c|c|}
\hline \multirow[b]{2}{*}{ Optimisation } & \multicolumn{3}{|c|}{ Release window } \\
\hline & $\begin{array}{l}\text { Mean [ms] } \\
(\mathrm{n}=1000)\end{array}$ & $\begin{array}{l}\%>10 m s \\
(n=1000)\end{array}$ & $\begin{array}{c}\%>29 \mathrm{~ms}^{+} \\
(\mathrm{n}=1000)\end{array}$ \\
\hline $\begin{array}{l}\text { Variability } 100 \% \\
\text { Torques } 100 \%\end{array}$ & $34 \pm 25$ & 69 & 65 \\
\hline $\begin{array}{l}\text { Variability } 100 \% \\
\text { Torques } 125 \%\end{array}$ & $40 \pm 25$ & 77 & 71 \\
\hline $\begin{array}{l}\text { Variability } 75 \% \\
\text { Torques } 100 \%\end{array}$ & $42 \pm 21$ & 84 & 79 \\
\hline $\begin{array}{l}\text { Variability } 75 \% \\
\text { Torques } 125 \%\end{array}$ & $49 \pm 20$ & 91 & 85 \\
\hline $\begin{array}{l}\text { Maximised } \\
\text { window }\end{array}$ & $14 \pm 30$ & 19 & 18 \\
\hline Gymnast & $7 \pm 13^{*}$ & $17^{*}$ & $8^{*}$ \\
\hline
\end{tabular}

* Numbers based on the 60 trials from Hiley et al. (2007) assuming similar release windows for the additional 40 unsuccessful Tkatchevs to the 20 analysed

$+29 \mathrm{~ms}$ was the mean release window of the 10 successful Tkatchevs 
Increasing the strength of the model and decreasing the amount of variability added to the joint angle time histories both increased the consistency of performance, to $77 \%$ and $84 \%$ respectively. When strength was increased and variability decreased by $25 \%$ together the most consistent performance was achieved, being $91 \%$ successful. None of the optimisations approached the bounds of the anatomical constraints.

The common feature of the optimum simulations was a slightly earlier phasing of the hip flexion and shoulder extension as the gymnast passed beneath the bar, an opening of the shoulder angle over a larger range close to release and a slightly later hip extension (Figure 4a). Although joint torques lay within the strength limits of the joint angle - angular velocity - torque profiles the optimum solution required larger shoulder extension torques since a larger shoulder flexion and extension angle was used compared with the gymnast performances (Figure $4 \mathrm{~b}$ ). This was achieved by the optimum simulation reaching limiting torque values at different times to the matching simulation. It is also noted that both the shoulder and hip torques were acting eccentrically towards the end of the giant circle (Figure 4b).

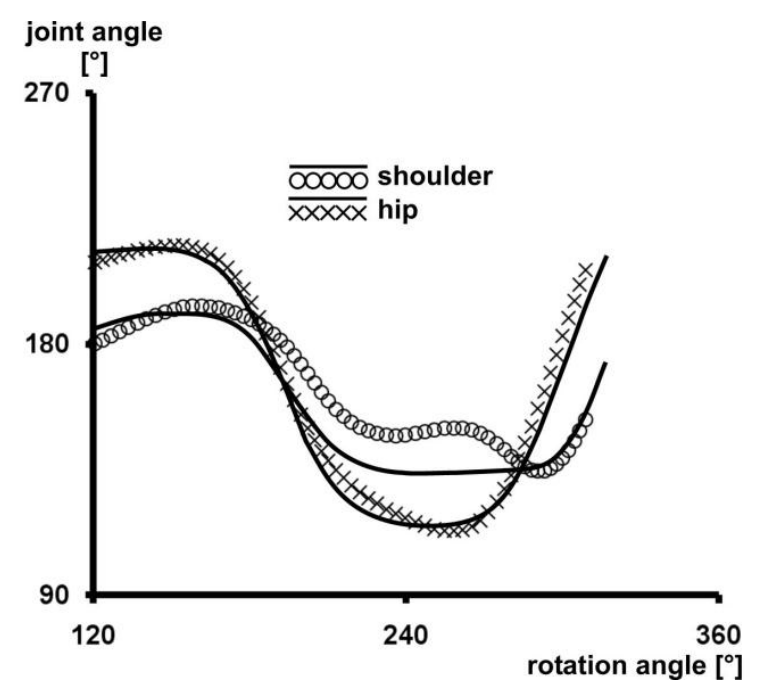

a

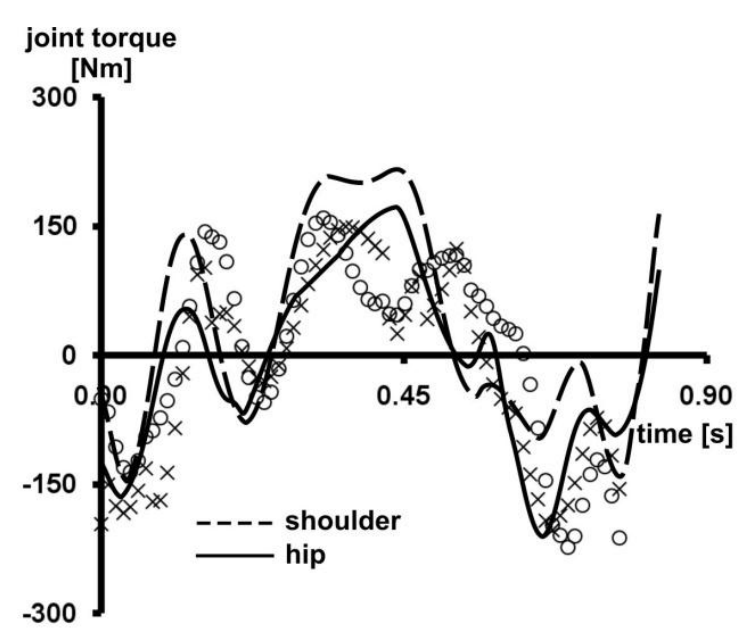

b

Figure 4. Optimum simulation (lines) robust to perturbations with a standard deviation of $12 \mathrm{~ms}$ and $2.3^{\circ}$ (a) joint angle time histories and (b) joint torques compared with time histories of matching simulation (symbols).

The final optimisation, which maximised the release window with no variability, produced a release window of $103 \mathrm{~ms}$. When this optimal solution was randomly perturbed in 1000 simulations the mean release window was $14 \mathrm{~ms}$ and only $19 \%$ of simulations produced a successful release window (Table 2).

\section{DISCUSSION}

The aim of this study was to investigate to what extent changes in technique, strength and flexibility can improve the consistency of the Tkatchev while including coordination variability based on gymnast performances. For the gymnast used in the present study releasing the bar earlier in the unsuccessful trials, as suggested by Holvoet et al. (2002), was not feasible since production of the correct amount of angular momentum did not coincide with the appropriate mass centre velocity (i.e. 
the gymnast had very small release windows, $<5 \mathrm{~ms}$, or no release window at all). In order to improve the consistency of performance the gymnast would be required to change his technique in the backward giant circle prior to release. Performing the hip flexion and shoulder extension under the bar slightly earlier, flexing the shoulder over a larger range and a slightly later hip extension prior to release in simulations resulted in improved consistency.

In the present study a more consistent performance, in terms of producing a suitably large release window to allow a successful Tkatchev, was achieved whilst maintaining realistic joint torques at the hip and shoulder. The largest increase in consistency was obtained by altering technique. The gymnast's technique only produced a successful performance $17 \%$ of the time (when considering all 60 gymnast trials); by modifying the technique the model was able to increase this to $69 \%$, a marked improvement. Further gains in consistency were achieved by reducing the amount of variability present in the joint angle time histories and by increasing the strength of the model. For the gymnast in the present study shoulder flexibility did not appear to play a significant role as none of the optimisations approached the bounds set by the gymnast's performances.

In the analysed performances the mean standard deviation of the start and end points of hip and shoulder flexion/extensions was $12 \mathrm{~ms}$ (Table 1). However, peak deviations from the mean were larger than this, over $30 \mathrm{~ms}$ (Figure 5). Using a random number generator with a normal distribution to add variability with a specified standard deviation produced a good match to the variability in the actual performances (Figure 5) both in terms of the mean deviation and the peak deviation. When the timing and angle variability is viewed within a joint angle time history it can be seen that the method used produces a comparable envelope of joint angle time histories when compared with the actual performances (Figure 6). Optimising without variability can produce a significantly larger release window (103 ms) than any of the analysed gymnast performances. However, when the solution was randomly perturbed in 1000 simulations the mean release window was $14 \mathrm{~ms}$ and only $19 \%$ resulted in a successful release window (Table 2 ). It is therefore important that optimisations should include those aspects of human movement (such as variability) which are likely to have a direct impact on the outcome.

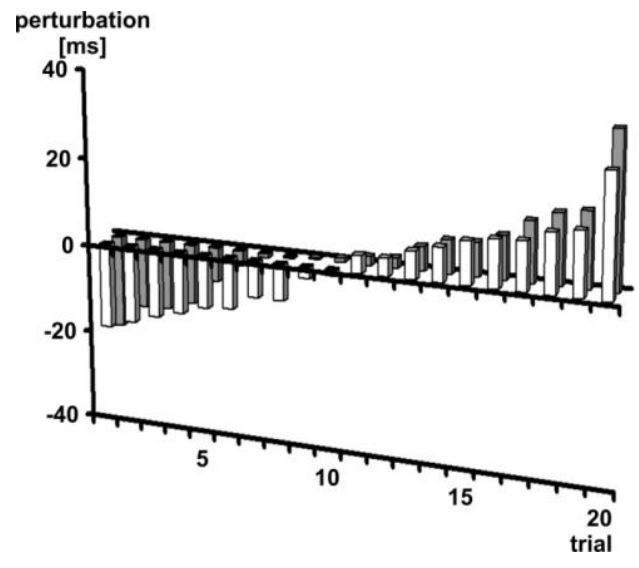

Figure 5. Timing variations about the mean at maximum shoulder flexion for the 20 actual performances (white) and 20 perturbations (standard deviation $12 \mathrm{~ms}$ ) from the random number generator (grey), arranged in ascending order. 


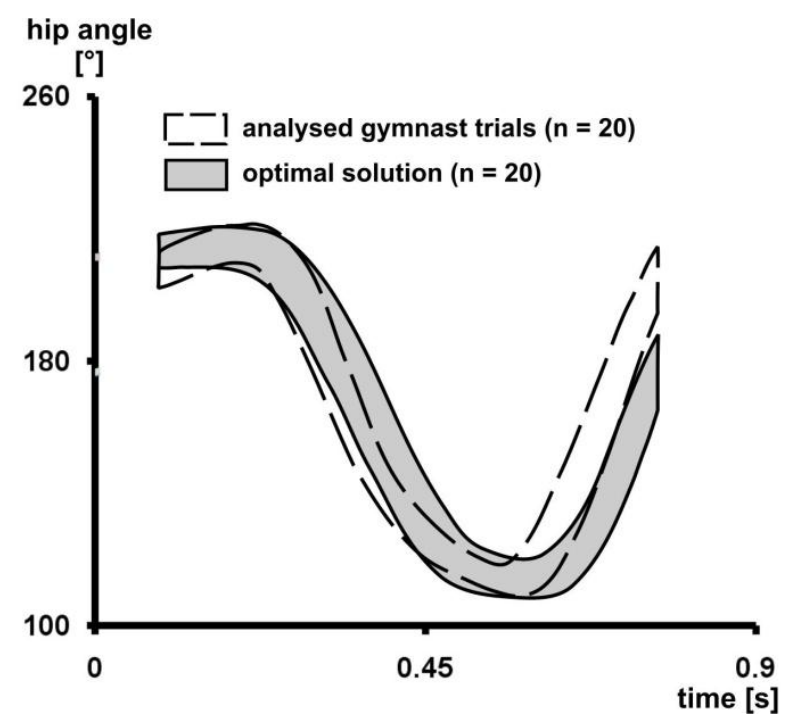

Figure 6. Envelopes containing the hip joint angle time histories obtained from (a) the 20 gymnast performances and (b) 20 perturbed simulations based on the optimal solution (standard deviations, $12 \mathrm{~ms}$ and $2.3^{\circ}$ ).

The study used a single subject design, but, this should not be viewed necessarily as a limitation. Although with other gymnasts there may be small changes in optimal technique and the amounts of improvement in consistency, it is expected that the pattern of improvement would remain similar. The method of obtaining the gymnast's variability in timings and angles relied on interpolation of quintic splines fit to experimental data. Given the frequency content of the joint angle time histories, sampling at a higher rate is unlikely to produce improved results. It is also noted that none of the optimisations were able to achieve a successful performance $100 \%$ of the time. Since it is unlikely that the gymnast could achieve a timing precision with a standard deviation much less than $9 \mathrm{~ms}$ and further increases in strength may also be unrealistic, it is believed that the function used to define the joint angle time histories may be too simple. A more sophisticated function that allows more rapid changes between flexion and extension might be expected to improve the level of consistency further, since changes in technique were shown to have the greatest effect on consistency (Table 2).

\section{CONCLUSION}

A method of optimising technique including variability based on human performances has been presented. The consistency of performance could be improved through changes to technique as well as increases in strength. While a specific gymnastics movement has been studied there are general implications for the study and understanding of human movement. The precision with which movement can be repeated has a marked effect upon consistency and success in a limiting movement. In optimisation studies it is necessary to consider issues of robustness to timing and angle variability and to base such analyses on measured levels of coordination precision. 


\section{Conflict of Interest Statement}

The authors wish to disclose that they have no financial or personal relationships with any people or organisations that could inappropriately influence this work.

\section{REFERENCES}

Brüggemann, G-P., Cheetham, P.J., Alp, Y. and Arampatzis, D. (1994). Approach to a biomechanical profile of dismounts and release-regrasp skills of the high bar. Journal of Applied Biomechanics, 10, 291-312.

Carroll, D.L. (2001). FORTRAN genetic algorithm driver. Downloaded from: http://cuaerospace.com/carroll.ga.html

Gervais, P. and Tally, F. (1993). The beat swing and mechanical descriptors of three horizontal bar release-regrasp skills. Journal of Applied Biomechanics, 9, 66-83.

Hiley, M.J. and Yeadon, M.R. (2003). Optimum technique for generating angular momentum in accelerated backward giant circles prior to a dismount. Journal of Applied Biomechanics, 19, 119-130.

Hiley, M.J. and Yeadon, M.R. (2005). The margin for error when releasing the asymmetric bars. Journal of Applied Biomechanics, 21, 223-235.

Hiley, M.J., Yeadon, M.R. and Buxton, E. (2007). Consistency of performance in the Tkatchev release and regrasp on high bar. Sports Biomechanics, 6, 119-128.

Holvoet, P., Lacouture, P. and Duboy, J. (2002). Practical use of airborne simulation in a release-regrasp skill on the high bar. Journal of Applied Biomechanics, 18, 332-344.

Kerwin, D.G., Yeadon, M.R. and Lee, S.C. (1990). Body configuration in multiple somersault high bar dismounts. International Journal of Sport Biomechanics, 6, 147-156.

King, M.A., Kong, P.W. and Yeadon, M.R. (2009). Determining effective subjectspecific strength levels for forward dives using computer simulation of recorded performances. Journal of Biomechanics, 42, 2672-2677.

King, M.A., and Yeadon, M.R. (2002). Determining subject-specific torque parameters for use in a torque driven simulation model of dynamic jumping. Journal of Applied Biomechanics, 18, 207-217.

Newell, K.M. and Corcos, D.M. (1993). Variability and Motor Control. Human Kinetics. Champaign, IL.

van Soest, A.J. and Casius, R.L.J. (2003). The merits of a parallel genetic algorithm in solving hard optimiszation problems. Journal of Biomedical Engineering, 125, 141-146.

Wilson, C., Yeadon, M.R. and King, M.A. (2007). Considerations that affect optimised simulation in a running jump for height. Journal of Biomechanics, 40 , 3155-3161. 
Wilson, C., Simpson, S.E., Van Emmerick, R.A. and Hamill, J. (2008). Coordination variability and skill development in expert triple jumpers. Sports Biomechanics, 7 , 2-9.

Wood, G.A. and Jennings, L.S. (1979). On the use of spline functions for data smoothing. Journal of Biomechanics, 12, 477-479.

Yeadon, M.R. (1990a). The simulation of aerial movement - I. The determination of orientation angles from film data. Journal of Biomechanics, 23, 59-66.

Yeadon, M.R. (1990b). The simulation of aerial movement - II. A mathematical inertia model of the human body. Journal of Biomechanics, 23, 67-74. 